\title{
Theoretical Study of the Movement of Cotton Seed Flow along the Contour of the Rib
}

\author{
Botir Mardonov1, Farkhod Ibrokhimov', Khamit Akhmetxodjayev², Khayrullo Sharipov², \\ Mukhammad Tojiboyev²
}

${ }^{1}$ Tashkent Institute of Textile and Light Industry, Tashkent, Uzbekistan

${ }^{2}$ Namangan Institute of Engineering and Technology, Namangan, Uzbekistan

Email: olimjon5008@mail.ru

How to cite this paper: Mardonov, B. Ibrokhimov, F., Akhmetxodjayev, K., Sharipov, K. and Tojiboyev, M. (2021) Theoretical Study of the Movement of Cotton Seed Flow along the Contour of the Rib. Engineering, 13, 526-535.

https://doi.org/10.4236/eng.2021.1310037

Received: January 12, 2021

Accepted: October 18, 2021

Published: October 21, 2021

Copyright (C) 2021 by author(s) and Scientific Research Publishing Inc. This work is licensed under the Creative Commons Attribution International License (CC BY 4.0).

http://creativecommons.org/licenses/by/4.0/

\begin{abstract}
Improving the efficiency of the process of separating fiber from cotton seeds by improving the working chamber of the gin is one of the important issues. The main way to increase the efficiency of sawn gin is to increase the fiber content in the mass of seeds in the working chamber with a uniform decrease in its density. This can be achieved by using grates with a concave working surface, which serves to move the seeds away from the rotating saws and accelerate the release of bare seeds from the working chamber. The article discusses the movement of seeds on the concave surface of the grate and determines the rational parameters of their working part. The article explores the proposed grate model, consisting of four geometric shapes, provides an analytical analysis of the geometric types. The dependence of the location of the last rectilinear part of the general contour on the shape of its convexity and concavity is determined. Cotton seeds move along the contour in the form of a stream. We assume that the thickness of the flow along the contour is constant and equal. We compose a unique equation of the flow in each section of the circuit. To determine the state of the flow, we denote its velocity, density and pressure in each section, respectively. Let us determine the flow motion along the contour with respect to the arc.
\end{abstract}

\section{Keywords}

Cotton, Saw Gin, Grates, Ginning, Fiber, Seeds, Concave Surface

\section{Introduction}

Currently, much attention is paid to increasing the output of value-added finished products, improving the cotton industry system based on the deep processing of cotton raw materials, lowering production costs and improving the 
quality of cotton products based on the re-equipment of cotton primary processing technology.

When solving these problems, increasing the efficiency of the ginning process by improving the working chamber of the gin is one of the important tasks.

Also, the main way to increase the efficiency of the saw gin is to increase the fiber content of the raw roller, accelerate the yield of bare seeds and a uniform decrease in its density [1] [2] [3] [4].

The rib-grate is one of the main parts of the working chamber of the saw gin. It serves as a free pass saw blades between the grates in the working chamber and the free output of the fibers engaged on the saw teeth after separation. Rib-grite are made by casting of cast iron grade SCh-15-32.

Working surfaces, processed on special machines, are reduced to a certain shape. The surface of the grate is hardened by he For normal operation of the saw blades, the working surface of the grate should be smooth and not rough, the distance between the grates in the working area should be $3 \pm 0.2 \mathrm{~mm}$, and in the lower part $4.5-5 \mathrm{~mm}$ at treatment [5] [6] [7] [8].

The number of grid-irons in the grate is one more than the number of saws on the shaft, two narrow grid-irons are installed in two extreme positions, and the rest with a normal width are intermediate. At the ginneries during the work of saw gins DP-130, the teeth of the saw cylinder, catching the fibers from the raw roller, pass between the grates and are separated from the seeds. The fibers hooked onto the saw teeth are separated by an air stream exiting the nozzle.

The surface of the grate of this gin is flat, the interaction of the raw roller and the saw cylinder is constant, the machine performance is low, energy consumption and damage are high.

Taking into account the above, we have proposed a grate with a concave profile for the rapid removal of bare seeds from the working chamber.

This rib-grate makes it possible to reduce friction with a saw cylinder and a raw roller, as a result of reducing damage to the fiber and seeds, as well as reducing energy consumption, accelerating the exit of bare seeds from the working chamber [8] [9] [10] [11] [12].

It has been proven that an increase in the productivity of gin is necessarily accompanied by an increase in the density of the seed roll. But the increase in productivity with increasing density occurs within certain limits, after the productivity decreases. This is due to a decrease in the speed of the seed roll under the influence of friction forces, it is determined that at a density of $550-600$ $\mathrm{kg} / \mathrm{m}^{3}$, the process completely stops, is the reason for a negative effect on the ginning process, a decrease in the productivity and quality of the fiber. To prevent this drawback, a more perfect study of the ginning process is required, the development of other methods for removing bare seeds from the roll box. The studies carried out to date in this area have not sufficiently investigated the issue of accelerating bare seeds from the roll box of the gin by improving the design of the grate during the ginning process [5] [13] [14].

The aim of the work is to increase the efficiency of ginning by improving the 
design of the grate of the saw gin stand.

Wedge-shaped depressions in adjacent grates form a tray. This tray, in the process of interaction of cotton with the saw in the contact zone, ensures their overturning and increased contact with the saw [15] [16] [17] [18].

The grate proposed by P. N. Tyutin [19], in the upper part it is made convex. This in turn leads to an increase in productivity, but to a decrease in seed and fiber quality.

The grate proposed by Sh. T. Ergashev [20], due to the better mesh of the fibers with the saw blade, is aimed at increasing productivity. The upper working surface of the grate is flat, both parts are made obliquely to each other. This ensures efficient movement of seeds on adjacent rotating saws across the width of the machine. In practice, these grates have not been used in production.

In the work of R. Sulaimanov [21], an algorithm for solving the problem of a mathematical model and dynamics of seed movement was obtained, which allows theoretically determining the optimal parameters of the distance between cantilever grates, grates and spacers when withdrawing additional seeds. On the basis of this, the distance between the saws has been reduced, and a new method and device for outputting additional seeds has been proposed.

\section{Simulation of Ginning Seeds on the Control Rib-Grate}

Suppose the grate outline consists of two circles and two straight lines. Cotton seeds move along the contour in the form of a stream. We assume that the thickness of the flow along the contour is constant and equal to $h_{0}$.

Let the initial grate contour be composed of an arc of a circle $A B$, its continuation of a straight line $\mathrm{BC}$, then an arc of a circle $\mathrm{CB}$ and a straight line $\mathrm{BE}$ (Figure 1).

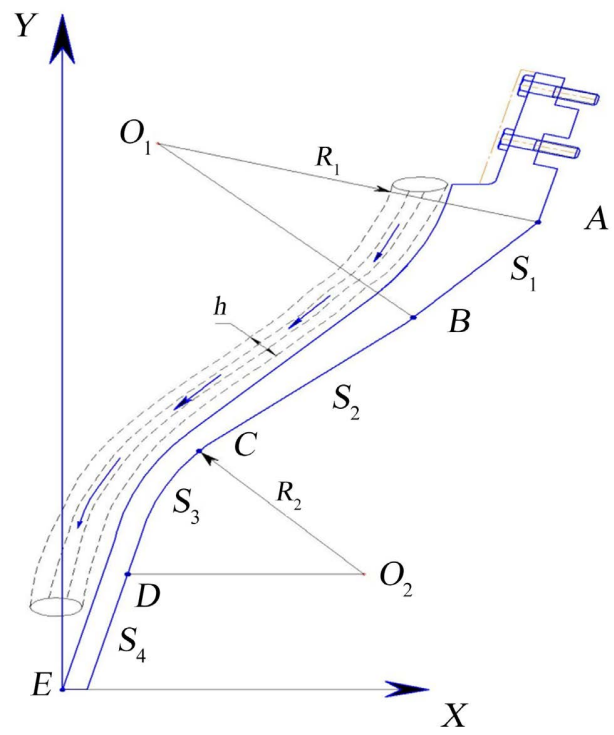

Figure 1. The movement pattern of the flow of cotton seeds along the contour of the rib-grate. $\mathrm{AB}, \mathrm{CD}$-arcs of circles, $\mathrm{BC}, \mathrm{DE}$ - straight lines. 
We compose a unique equation of the flow in each section of the circuit. To determine the state of the flow, we denote its velocity, density and pressure in each section, respectively, $\left(v_{1}, \rho_{1}, P_{1}\right),\left(v_{2}, \rho_{2}, P_{2}\right),\left(v_{3}, \rho_{3}, P_{3}\right),\left(v_{4}, \rho_{4}, P_{4}\right)$. Let us determine the flow motion along the contour with respect to the arc. We calculate the length of each arc from point $\mathrm{A}$ and let the length of each section consist of $0<s<s_{a}$ (section $\left.\mathrm{AB}\right), s_{01}<s_{0}+s_{1}$ (section $\left.\mathrm{BC}\right), s_{0}+s_{1}<s_{0}+s_{1}+s_{02}$ (section $\mathrm{CB}$ ) and $s_{01}+s_{1}+s_{0}<s<s_{01}+s_{1}+s_{0}+s_{2}$ (section DE). We write the Euler equation for each section:

$$
\begin{gathered}
\rho_{1} v_{1} \frac{\partial v_{1}}{\partial s}=-\frac{\partial p_{1}}{\partial s}+\rho_{1} g\left[\sin \left(\alpha_{00}+s / R\right)+f \cos \left(\alpha_{00}+s / R\right)\right] \\
-f \rho_{1} \frac{v_{1}^{2}}{R_{1}}, 0<s<s_{01} \\
\rho_{2} v_{2} \frac{\partial v_{2}}{\partial s}=-\frac{\partial p_{2}}{\partial s}+\rho_{2} g\left[\sin \left(\alpha_{00}+\alpha_{01}\right)\right. \\
\left.-f \cos \left(\alpha_{00}+\alpha_{02}\right)\right], s_{01}<s<s_{01}+s_{1} \\
\rho_{3} v_{3} \frac{\partial v_{3}}{\partial s}=-\frac{\partial p_{3}}{\partial s}+\rho_{3} g\left[\sin \left(\alpha_{00}+s / R-s_{1} / R\right)-f \cos \left(\alpha_{00}+s / R-s_{1} / R\right)\right] \\
-f \frac{v_{3}^{2}}{R_{2}}, s_{01}+s_{1}<s<s_{01}+s_{1}+s_{02} \\
\rho_{4} v_{4} \frac{\partial v_{4}}{\partial \mathrm{s}}=-\frac{\partial p_{4}}{\partial s}+\rho_{4} g\left[\sin \left(\alpha_{00}+\alpha_{01}+\alpha_{02}\right)\right. \\
\left.-f \cos \left(\alpha_{00}+\alpha_{01}+\alpha_{02}\right)\right], s_{01}+s_{1}+s_{02}<s<s_{01}+s_{1}+s_{02}+s_{2}
\end{gathered}
$$

Here, $f-$ is the coefficient of friction between the flow and the rib-grate, $R_{1}, R_{2}$ are the radii of the circles.

The coordinate origin is set at point $\mathrm{E}$, the axis ox is directed to the right, the axis oy is directed up.

The coordinates of points $\mathrm{A}, \mathrm{B}, \mathrm{C}, \mathrm{D}$ and $\mathrm{E}$, the angles $\alpha_{01}, \alpha_{02}$, as well as the radii $R_{1}$ and $R_{2}$ have a certain value, for Equations (1)-(4) the lengths are determined by the following formulas.

$$
\begin{gathered}
s_{01}=\alpha_{01} R_{1}, \quad s_{1}=\sqrt{\left(x_{1}-x_{2}\right)^{2}+\left(y_{1}-y_{2}\right)^{2}}, \quad s_{02}=\alpha_{02} R_{2}, \\
s_{2}=\sqrt{\left(x_{2}-x_{3}\right)^{2}+\left(y_{3}-y_{2}\right)^{2}}
\end{gathered}
$$

In Equations (1)-(4), three unknowns participate $P_{i}, \rho_{i}, v_{i}, i=1,2,3$, to describe them in relation to one unknown, more precisely in relation to $k v_{i}$, we use these two conditions:

The flow is stationary, then the following condition must be fulfilled

$$
\rho_{i} \cdot v_{i} \cdot h \cdot L=Q_{0}
$$

Here, $Q$-is the productivity of one grate for seed flow, $L$ is the width of the grate.

From Equation (5) we express the density in terms of speed:

$$
\rho_{i}=\frac{Q_{0}}{v_{i} \cdot h_{i} \cdot L}
$$


We accept the seed flow as a conjugate medium; therefore, the equation of state of the medium will be appropriate.

$$
\rho_{i}=\rho_{0}\left[1+A\left(p_{i}-p_{0}\right)\right]
$$

Here, $\rho_{0}, p_{0}$-is the density and pressure in the stream before feeding to the grate; $A$-experimentally determined physical quantity inversely proportional to the stiffness of the medium consisting of the mass of seeds.

To determine the flux density before feeding, we define the volume between any grates and two gear discs adjacent to them.

In accordance with Figure 2, this volume is calculated by the following formula.

$$
V=\left[\pi \beta R^{2} / 2-\left(S_{1}+S_{2}+S_{3}+S_{4}\right)\right] L
$$

Here, $S$ - the area of the triangle.

$S_{1}=0.5 A B \sin \beta_{1}, \quad S_{2}=0.5 B C \sin \beta_{2}, \quad S_{3}=0.5 C D \sin \beta_{3}, \quad S_{4}=0.5 D E \sin \beta_{4}$,

$$
\begin{gathered}
\beta_{1}=\arccos \sqrt{\frac{R^{2}+R_{1}^{2}-A B^{2}}{R_{1} R}}, \quad \beta_{2}=\arccos \sqrt{\frac{R_{2}^{2}+R_{1}^{2}-B C^{2}}{R_{1} R_{2}}}, \\
\beta_{3}=\arccos \sqrt{\frac{R_{3}^{2}+R_{2}^{2}-C D^{2}}{R_{2} R_{3}}}, \\
\beta_{4}=\arccos \sqrt{\frac{R^{2}+R_{3}^{2}-D E^{2}}{R_{3} R}}, \quad \beta=\beta_{1}+\beta_{2}+\beta_{3}+\beta_{4}
\end{gathered}
$$

In the calculations we take, $A B=s_{01}, B C=s_{1}, C D=s_{02}, D E=s_{2}$.

Let's say the volume $V$ is completely filled with seeds of pieces with the same mass $m$.

Then the total mass of the volume is $M=N \cdot m$, and its density can approximately be calculated by the following formula.

$$
\rho_{0} \approx N \cdot m / V
$$

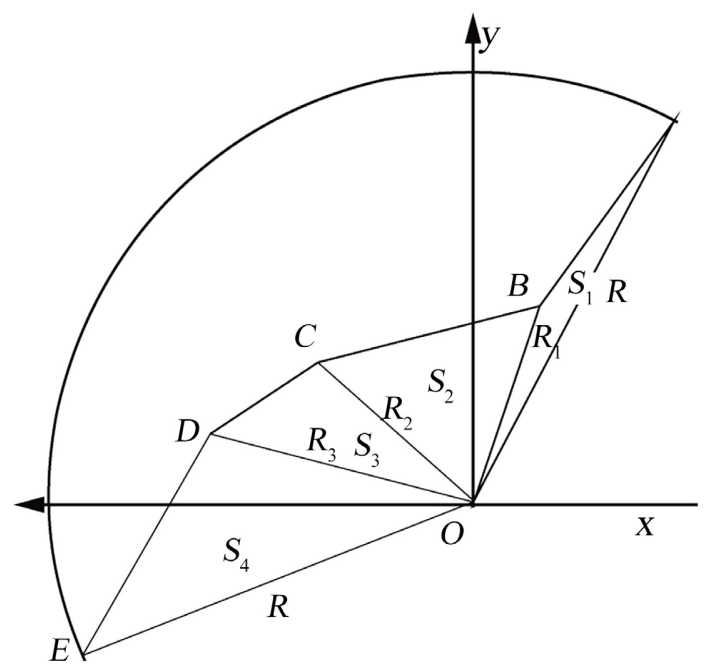

Figure 2. The calculated contour of the rib-grate. 
Using formula (7) we find the relationship between pressure and density.

$$
v_{i}=\frac{Q_{0}}{\rho_{0} h_{i} L\left[1+A\left(p_{i}-p_{0}\right)\right]}
$$

From Equation (8) we determine the derivative $\frac{\partial p_{i}}{\partial s}$

$$
\frac{\partial p_{i}}{\partial s}=-\frac{v_{0}}{v_{i}^{2} A} \frac{\partial v_{i}}{\partial s}
$$

Using formulas (6) and (9), Equations (1)-(4) lead to the following forms with respect to velocities $v_{i}$.

$$
\begin{gathered}
\frac{\partial v_{1}}{\partial s}=\frac{g v_{1}}{v_{1}^{2}-c^{2}}\left(\sin \frac{s}{R_{1}}+f \cos \frac{s}{R_{1}}\right)-f \frac{v_{1}^{3}}{v_{1}^{2}-c^{2}} \frac{1}{R_{1}}, \quad s_{00}<s<s_{01} \\
\frac{\partial v_{2}}{\partial s}=\frac{g v_{2}}{v_{2}^{2}-c^{2}}\left(\sin s_{01} / R-f \cos s_{01} / R\right), \quad s_{01}<s<s_{01}+s_{1} \\
\frac{\partial v_{3}}{\partial s}=\frac{g v_{3}}{\left(v_{3}^{2}-c^{2}\right) v_{3}}\left(\sin \frac{s}{R_{2}}-f \cos \frac{s}{R_{2}}\right) \\
\quad-f \frac{v_{3}^{3}}{v_{3}^{2}-c^{2}} \frac{1}{R}, \quad s_{01}+s_{1}<s<s_{01}+s_{1}+s_{02} \\
\frac{\partial v_{4}}{\partial s}=\frac{g v_{4}}{\left(v_{4}^{2}-c^{2}\right) v_{4}}\left(\sin s_{02} / R\right. \\
\left.-f \cos s_{02} / R\right), \quad s_{01}+s_{1}+s_{02}<s<s_{0}+s_{1}+s_{0}+s_{2}
\end{gathered}
$$

Here, $c=\sqrt{1 / A \rho_{0}}$.

Equations (10)-(13) are nonlinear, we integrate numerically under the following conditions:

$$
\begin{gathered}
v_{1}=v_{10} \text { at } s=s_{00} \\
v_{2}\left(s_{01}\right)=v_{1}\left(s_{01}\right) \text { at } s=s_{01} \\
v_{3}\left(s_{1}+s_{01}\right)=v_{2}\left(s_{1}+s_{0}\right) \text { at } s=s_{1}+s_{01} \\
v_{4}\left(s_{1}+s_{01}+s_{02}\right)=v_{3}\left(s_{1}+s_{01}+s_{02}\right) \text { at } s=s_{1}+s_{01}+s_{02}
\end{gathered}
$$

\section{Analysis of the Model for Various Values of the Parameters of the Rib-Grate}

The following values are accepted in the calculations

$$
\begin{aligned}
& L=0.16, R_{1}=0.107, R_{2}=0.1145, s_{01}=0.07098120639, \\
& s_{1}=0.07098120639, s_{2}=0.07604794422, s_{3}=0.07266443270, \\
& s_{4}=0.082050115661, s_{0}=0.3017437399, \\
& L=0.00818 \mathrm{~m}, m=0.025 \times 10^{-3} \mathrm{~kg}, N=250, f=0.2
\end{aligned}
$$

As a result, the following results were obtained, in Figure 2 shows the calculated contour of the rib-grate. 


$$
\begin{aligned}
& \beta_{1}=\alpha_{1}=0.3628444444, \alpha_{2}=0.6977777778, \alpha_{3}=0.5378122222, \\
& \alpha_{4}=0.2485833333, \alpha_{0}=1.847017777, \beta=\alpha_{0}=105.8799999, \\
& v=0.00007972317841 \mathrm{~m}^{3}, \rho_{0}=31.35850890 \mathrm{~kg} / \mathrm{m}^{3}
\end{aligned}
$$

In Figure 3 and Figure 4 show plots of the distribution of the flow rate of the mass of seeds along the contour for various values of the angle and parameter, indicating its mechanical properties.

It can be seen from the graph that the flow velocity along the circuit is close to a linear regularity, and with increasing parameter $A$, the flow velocity decreases upon exiting the circuit. It is also seen that with increasing angle $\beta_{4}$ practically does not affect the distribution of speed.

The parameter can significantly affect the change in flux density (Figure 4) and with its increase, an increase in density, the formation of the mass of compacted seeds on the grate circuit is possible [12] [22] [23].
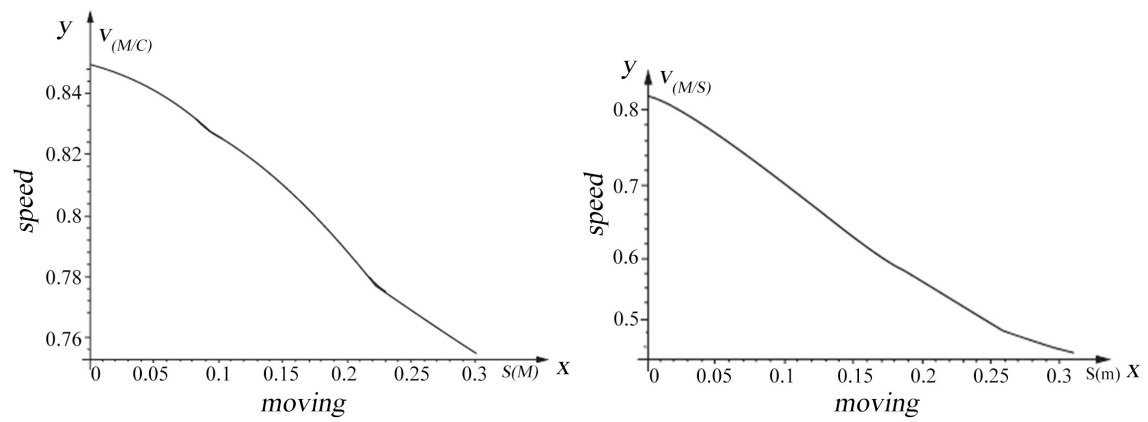

Figure 3. Graphs of the distribution of seed flow velocity along the contour for two values of the angle $\beta_{4}$ and parameter $A . A=0.001 \mathrm{~Pa}^{-1} ; A=0.005 \mathrm{~Pa}^{-1} ; \beta_{4}=50^{\circ}$.
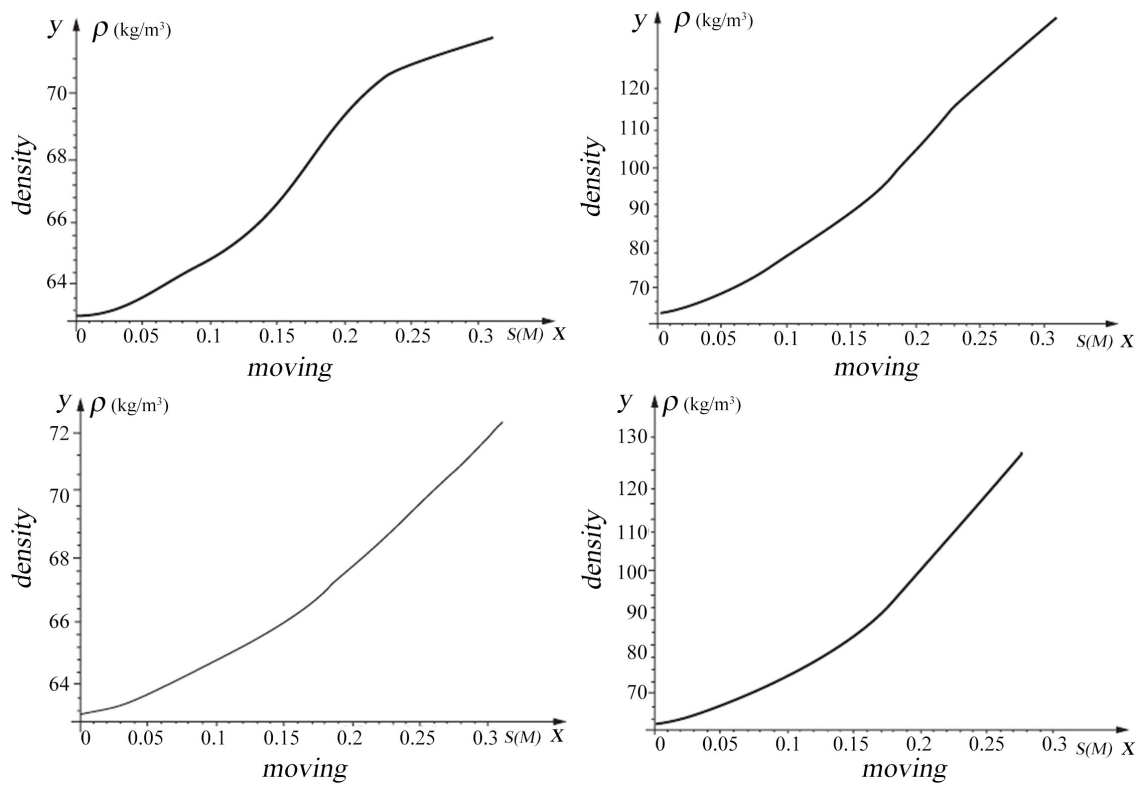

Figure 4. Graphs of the density distribution $\rho\left(\mathrm{kg} / \mathrm{m}^{3}\right)$ of the seed flow along the contour for two values of the angle and parameter. $A=0.001 \mathrm{~Pa}^{-1} ; A=0.005 \mathrm{~Pa}^{-1} ; \beta_{4}=50^{\circ}$, $A=0.001 \mathrm{~Pa}^{-1} ; A=0.005 \mathrm{~Pa}^{-1} ; \beta_{4}=80^{\circ}$. 


\section{Findings}

1) A grate model consisting of four geometric forms is proposed, an analytical analysis of geometric types is presented. The dependence of the location of the last rectilinear part of the contour of the general contour on the shape of its convexity and concavity is determined.

2) A system of differential equations has been obtained to determine the law of the distribution of seed flow velocity along the contours of the proposed grate, using the law of conservation of mass and the equation of state of the medium. From the analysis of the law of the distribution of flow velocities, we observed the proximity of its distribution along the contour to a straight line, as well as a decrease in velocity along the contour.

3) The regularity of increasing the density of seed flow along the contour was determined, the possibility of increasing the density with increasing parameter (that is, with decreasing rigidity of the medium of the mass of seeds) was observed. This shows the probability of the formation of a high degree of density on the contour of the grate and in some cases the appearance of fixed zones of the medium.

\section{Conflicts of Interest}

The authors declare no conflicts of interest regarding the publication of this paper.

\section{References}

[1] Sarimsakov, A., Karimov, A.I. and Murodov, R. (2012) Static Calculation of Processes in the Roll Box of the Saw Gin. Problems of Mechanics, No. 2, 78-85.

[2] Akhmedkhodjaev, Kh.T., Karimov, A.I., Tojiboyev, M. and Sharipov, H. (2013) Determination of the Static Balance of Forces Affecting Seeds Moving along Grates with a Convex Profile. Scientific and Technical Journal of the Ferghana Polytechnic Institute, No. 2, 36-40.

[3] Akhmedkhodjaev, Kh.T., Karimov, A.I., Tojiboyev, M. and Sharipov, H. (2018) Theoretical Analysis of the Movement of Seeds along the Grate with a Convex Profile. Scientific and Technical Journal of the Ferghana Polytechnic Institute, No. 3, 59-63.

[4] Karimov, A.I., Azizov, Sh. and Ismanov, M. (2014) Mathematical Modeling of the Technological Processes Original Processing of Cotton. International Journal of Innovation and Applied Studies, 6, 28-39. http://www.ijias.issr-journals.org

[5] Akramjon, S., Rustam, M., Akmal, U. and Dilmurat, K. (2018) Movement Differential Equation of Seed Roller Which Has Been Installed Stake Accelerator on the Roll Box of Gin Machine. Engineering, 10, 521-529.

https://doi.org/10.4236/eng.2018.108038

[6] Akmal, U., Khamit, A., Akramjon, S. and Muazzam, K. (2018) The Saw Gin Stand with Adjustable Movement of the Roll Box. Engineering, 10, 486-494. https://doi.org/10.4236/eng.2018.108034

[7] Khamit, A., Akmal, U. and Kamola, O. (2019) Investigation of the Ginning Process on DП Series Saw Gin Stands. Engineering, 11, 523-530. 
https://www.scirp.org/journal/eng https://doi.org/10.4236/eng.2019.118036

[8] Sharipov, K., Akhmetxodjayev, K. and Mardonov, B. (2019) Theoretical Studies of the Movement of a Single Sustem along Grates with a Conceve Gin Profile. The Problèmes of Mexanical, 4, 55-58.

[9] Mardonov, B., Sharipov, K. and Akhmetxodjayev, K. (2019) Study of the Law of Motion of a Single Seed on the Rib. The Problèmes of Textile, 4, 42-50.

[10] Sharipov, K., Akhmetxodjayev, K. and Tadjibayev, M. (2019) The Mathematical Model of Seed Movement on a Concave Profile Rib of Saw Jin Stand. Scientific and Technical Journal of Namangan Institute of Engineering and Technology, 4, 19-29.

[11] Sharipov, K., Akhmetxodjayev, K. and Tadjibayev, M. (2019) Practical and Theoretical Study of the Process of Separating Seeds from Advanced Gin. Scientific and Technical Journal of Namangan Institute of Engineering and Technology, 3, 14-19.

[12] Sharipov, K., Akhmetxodjayev, K., Tadjibayev, M. and Sarimsakov, O. (2020) The Mathematical Model of Seed Movement on a Concave Profile Rib. Engineering, 12, 216-227. https://www.scirp.org/journal/eng https://doi.org/10.4236/eng.2020.123017

[13] Sarimsakov, A.U., Kurbonov, D.M. and Halikov, Sh.H. (2019) The Study of the Crucible Rotation of Raw Materials with a Practical Way. Scientific and Practical Journal "Economy and Society", No. 12, 67-72.

https://www.researchgate.net/publication/348309442_Study_of_the_crucible_of_rot ation_of_raw_materials_with_a_practical_way

[14] Umarov, A.A., Kurbanov, D.M., Akhmedkhodjaev, Kh.T. and Ergashev, J.F. (2020) Improving the Roll Box of the Saw Gin. The American Journal of Engineering and Technology, 2, 50-53. https://usajournalshub.com/index.php/tajet/article/view/1444

[15] Umarov, A.A., Kurbanov, D.M., Akhmedkhodjaev, Kh.T. and Kenjaeva, M.I. (2020) Saw Gin Accelerator. International Journal of Academic Engineering Research, 4, 59-63. http://ijeais.org/wp-content/uploads/2020/11/IJAER201110.pdf

[16] Umarov, A.A., Ortikova, K., Sarimsakov, A.U. and Kurbanov, D. (2020) Analysis of the Control of Manual and Automatic Regulation of the Saw Gin Feed Rollers. Scientific Journal “Universum: Technical Sciences”. Part 2, 9, 22-26. https://7universum.com/ru/tech/archive/item/10693

[17] Rejapova, N., Mutalov, M. and Sarimsakov, O. (2020) To Study of the Movement and Throughput of Cotton inside Pneumatic Conveying Pipeline. International Journal of Research, 7, 1018-1024. https://journals.pen2print.org/index.php/ijr/article/view/19931/19544

[18] Rejapova, N., Abdusattorov, B. and Sarimsakov, O. (2020) Training on the Pneumatic Work with Petroleum Patients within the Preliminary and Practical Way. International Journal of Psychosocial Rehabilitation, 24, 6416-6426. https://www.psychosocial.com/article/PR2020451/26695 https://doi.org/10.37200/IJPR/V24I4/PR2020451

[19] Tyutin, P.N. (1981) Author's Certificate. 23.10.81. Bulletin No. 39.

[20] Ergashev, Sh.T. (1989) Author’s Certificate. 07.10.89. Bulletin No. 37.

[21] Sulaimanov, R.Sh. (2006) Development of a Roll Box with Improved Ejaculation during Careful Ginning of Seed Cotton. The Dissertation on Competition of a Scientific Degree of the Candidate of Technical Sciences. Tashkent.

[22] Abdukarimovich, M.O., Ibragimovich, A.K. and Sharipjanovich, S.O. (2018) Designing a New Design of a Loading Cylinder for Pneumo-Mechanical Spinning 
Machines. Engineering, 10, 345-356. https://doi.org/10.4236/eng.2018.106025

[23] Sarimsakov, O.I., Tursunov, N. and Rajapova, B. (2020) Mardonov. The Study of the Movement of the Aero Mixture through the Pipeline during Pneumatic Transportation. Journal of Advanced Research in Dynamical and Control Systems, 12, 1287-1297. https://doi.org/10.5373/JARDCS/V12SP4/20201605 